\title{
Photodynamics of Fe complexes: Variation with number of NHC functions
}

\author{
Aleksej Friedrich ${ }^{1, *}$, Peter Zimmer ${ }^{2}$, Matthias Bauer $^{2}$, and Stefan Lochbrunner ${ }^{1}$ \\ ${ }^{1}$ Institute of Physics, University of Rostock, Albert-Einstein-Str. 23, 18059 Rostock, Germany \\ ${ }^{2}$ Department Chemie, Universität Paderborn, Warburger Straße 100, 33098 Paderborn, Germany
}

\begin{abstract}
Ultrafast spectroscopy on a series of Fe(II) complexes finds an increase of the ${ }^{3}$ MLCT lifetime with increasing number of N-heterocyclic carbene $(\mathrm{NHC})$ donor functions revealing a promising route for the design of Fe photosensitizers.
\end{abstract}

\section{Introduction}

Currently intense research is performed to develop environmentally friendly strategies based on photocatalysis for the generation of solar fuels and high-value chemicals. Several successful approaches have been already demonstrated [1]. However, most of them make use of photosensitizers containing metals like ruthenium, iridium, or platinum. Replacing these noble metals by inexpensive, earth abundant and potentially non-toxic metals is highly desirable. One attractive candidate for this propose is iron. However, $\mathrm{Fe}$ complexes suffer from short lifetimes of their metal-to-ligand charge transfer (MLCT) states which typically provide the active electrons and are crucial for the photocatalytic performance. Several strategies were applied in recent years to extend their lifetime, e.g. by introducing strong sigma-donating N-heterocyclic carbene (NHC) ligands like imidazolydene or benzimidazolydene functions to destabilize excited metal centred (MC) states and to reduce the deactivation of the ${ }^{3} \mathrm{MLCT}$ states by the MC states.

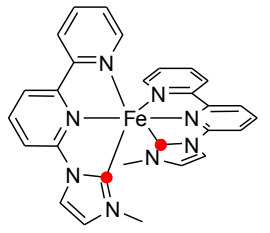

1

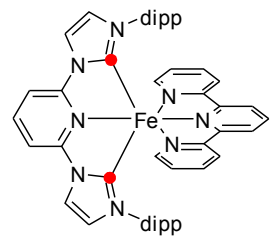

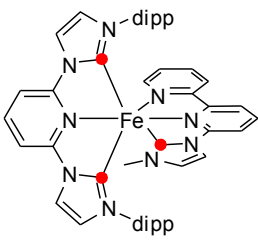

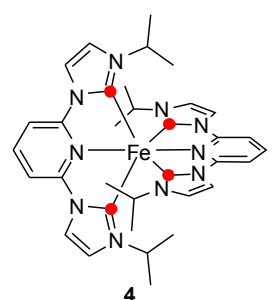

4

Fig. 1. Investigated complexes (with dipp = diisopropylphenyl). The NHC functions are indicated by red dots.

\footnotetext{
* Corresponding author: aleksej.friedrich@,uni-rostock.de
} 
Here we investigate the impact of the number of NHC functions on the ${ }^{3}$ MLCT lifetime by ultrafast absorption spectroscopy. To this end the complexes $\left[\mathrm{Fe}[\mathrm{L} 1]_{2}\right]\left[\mathrm{PF}_{6}\right]_{2}(\mathbf{1}, \mathrm{L} 1=1$ (2,2'-bipyridyl)-3-methylimidazol-2-ylidene), [Fe[L2]terpy][BPh $]_{2}(\mathbf{2}$, terpy $=$ terpyridine, L2 = 2,6-bis[3-(2,6-diisopropyl-phenyl)imidazol-2-ylidene]pyridine), [Fe[L1][L2]][PF6 $]_{2}$ (3), and $\left[\mathrm{Fe}[\mathrm{L} 3]_{2}\right]\left[\mathrm{BPh}_{4}\right]_{2}$ (4, L3 = 2,6-bis[3-isopropylimidazol-2-ylidene]pyridine) with two to four NHC functions are studied (see Fig. 1).

\section{Experimental results and discussion}

Transient absorption spectra were recorded with a time resolution of $100 \mathrm{fs}$ by a pumpprobe setup based on a non-collinear optical parametric amplifier (NOPA) for excitation and a white light continuum for probing. Fig. 2 shows the evolution of the absorption change $\Delta \mathrm{A}$ for the complexes 1 (left) and 3 (right) in acetonitrile after optical excitation at $550 \mathrm{~nm}$
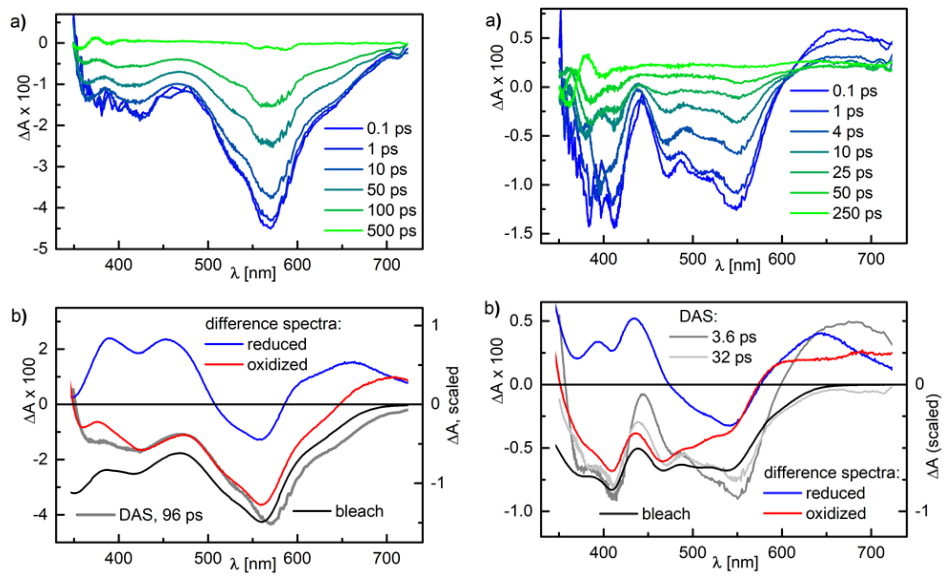

Fig. 2. a) Transient absorption spectra of $\mathbf{1}$ (left) and $\mathbf{3}$ (right). b) Decay associated amplitude spectra (DAS, grey line, left scale) of the exponential decay with the time constant of 96 ps. The DAS is compared to the inverted ground state absorption reflecting the excitation induced bleach (black line) and to the difference spectra between the reduced respectively oxidized form and the original ground state.

The transient spectra of complex 1 are dominated by a negative band with a spectral shape similar to the stationary absorption indicating that bleach of the ground state is responsible for most of the signal. The dynamics can be well fitted by a monoexponential decay with a time constant of $96 \mathrm{ps}$. The decay associated spectrum (DAS), which is in the lower graph compared to the bleach and the difference spectra of the reduced and oxidized form, disagrees with the difference spectrum of the reduced species while it seems to resemble the spectrum of the oxidized form at least in the green and blue spectral region. The similarities reflect the fact that in both cases an electron is removed from the metal based HOMO. Optical excitation of the Fe complexes should populate a MLCT state, since the LUMO is located at the ligands. MLCT states, however, exhibit typically an excited state absorption (ESA) on the red side of the ground state bleach, which is missing here. This points to the population of a metal centred (MC) state and the following relaxation scenario: After optical excitation of the singlet ${ }^{1}$ MLCT state, very fast intersystem crossing (ISC) to the ${ }^{3}$ MLCT state and internal conversion to the MC state occur within the time 
resolution of our pump-probe measurements. Then the $\mathrm{MC}$ state relaxes back to the ground state with a lifetime of 96 ps [2]. This kind of scenario was already reported for similar Fe complexes [3].

The transient absorption of complex 3 containing three Fe-carbene groups is again dominated by the ground state bleach and its recovery (see Fig. 2). However, the recovery is substantially faster and an additional ESA component is observed at wavelengths above $620 \mathrm{~nm}$. In this spectral region the transient absorption spectrum has some similarities with the difference spectrum of the reduced form of the complex. This indicates that the ESA band is related to an excess electron on the ligand and points to the involvement of charge transfer states. The dynamics can be fitted with a biexponential decay, the time constants being $3.6 \mathrm{ps}$ and $32 \mathrm{ps}$. The DAS of the $32 \mathrm{ps}$ decay resembles the inverse of the original absorption spectrum and reflects the return to the electronic ground state. The $3.6 \mathrm{ps}$ component contains the ESA band in the red which is ascribed to charge transfer contributions [2]. The $3.6 \mathrm{ps}$ component is therefore assigned to the depopulation of the ${ }^{3} \mathrm{MLCT}$ state to the MC state which relaxes subsequently back to the electronic ground state within 32 ps.

The transient spectra of the tetra-carbene complex 4 show also an ESA at the red side of the ground state bleach. The absorption change decays monoexponentially with a time constant of 8.1 ps and the DAS represents a superposition of the ground state bleach and the ESA band. Because of the ESA feature we attribute the observed dynamics to the depopulation of the ${ }^{3} \mathrm{MLCT}$ state. Accordingly it has a lifetime of 8.1 ps while the lifetime of the subsequently populated MC state is too short to accumulate significant population in this state and to cause transient signatures which can be assigned to the MC state.

\section{Conclusions}

The results point to a sequential relaxation pathway involving MLCT and MC states. First, ISC leads from the optically populated ${ }^{1}$ MLCT state extremely fast to the ${ }^{3}$ MLCT state. The lifetime of the latter increases with increasing number of NHC functions from below our time resolution in $\mathbf{1}$ and $\mathbf{2}$ to $3.6 \mathrm{ps}$ in $\mathbf{3}$ and then to $8.1 \mathrm{ps}$ in $\mathbf{4}$. In parallel the lifetime of the subsequently populated MC state decreases from the $100 \mathrm{ps}$ regime to below $10 \mathrm{ps}$. These findings can be explained by the destabilization of the MC state due to the NHC groups which reduces the coupling to the ${ }^{3}$ MLCT state and increases the one to the ground state. Accordingly, the strategy of inserting NHC functions seems to be promising for the design of Fe photosensitizers with sufficiently long lifetimes of the photocatalytically important ${ }^{3}$ MLCT states.

Financial support by the Deutsche Forschungsgemeinschaft (DFG) via the research unit FOR1405 (project TP5) and the collaborative research center SFB 652 is gratefully acknowledged.

\section{References}

1. H. Junge, N. Rockstroh, S. Fischer, A. Brückner, R. Ludwig, S. Lochbrunner, O. Kühn, and M. Beller, Inorganics 5, 14 (2017).

2. P. Zimmer, L. Burkhardt, A. Friedrich, J. Steube, A. Neuba, R. Schepper, P. Müller, U. Flörke, M. Huber, S. Lochbrunner, and M. Bauer, Inorg. Chem. 57, 360 (2018).

3. Y. Liu, T. Harlang, S. E. Canton, P. Chábera, K. Suárez-Alcántara, A. Fleckhaus, D. A. Vithanage, E. Göransson, A. Corani, R. Lomoth, et al., Chem. Commun. 49, 6412 (2013). 